\title{
Vector boson plus heavy flavor jets and constraints to PDFs
}

\author{
J. P. Fernández ${ }^{* \dagger}$ \\ CIEMAT \\ E-mail: juanpablo.fernandez@ciemat.es
}

\begin{abstract}
Measurements of the associated production of vector bosons, $\mathrm{W}$ or $\mathrm{Z}$ (decaying to one or two leptons), and jets originating from heavy-flavour quarks are reported using data corresponding to integrated luminosities of 19.7 (35.7) $\mathrm{fb}^{-1}$, at centre-of-mass energies of 8 (13) TeV collected by the CMS detector at the CERN LHC. Total and differential cross sections as a function of several kinematic observables are measured and compared with several theoretical predictions. The production of a $\mathrm{Z}$ boson associated with a c-quark allows probing and constraining the charm quark content of the proton and may give insight into the existence of an intrinsic charm quark component inside the proton. The production of a $\mathrm{W}$ boson associated with a c-quark allows probing and constraining the strange quark content of the proton.
\end{abstract}

European Physical Society Conference on High Energy Physics - EPS-HEP 2019,

July 10-17, 2019

Ghent (Belgium)

\footnotetext{
* Speaker.

${ }^{\dagger}$ On behalf of CMS Collaboration
} 


\section{Introduction}

The CERN LHC has delivered a large sample of pp collisions containing events with a vector boson $(\mathrm{V})$ accompanied by one or more jets (V+jets) originating from heavy-flavour (HF) quarks. The V+jets events can be used to study specific predictions of the standard model (SM). The study of the associated production of a W (Z) boson with jets from a charm quark at the LHC (hereafter referred to as $\mathrm{W}+\mathrm{c}$ and $\mathrm{Z}+\mathrm{c}$ production) provides direct access to the strange (charm) quark content of the proton at an energy scale of the order of the vector boson mass. The differential cross section as a function of the pseudorapidity of the lepton from the $\mathrm{W}$ decay is used in a quantum chromodynamics (QCD) analysis, together with other available measurements sensitive to the strange PDF, to extract the strange quark content of the proton. New Parton Distribution Functions (PDF) sets have been recently released [1] where the charm PDF is no longer assumed to be perturbatively generated through pair production from gluons and light quarks, but it is parameterized and determined along with the light quark and gluon PDFs. The main effect of this so called intrinsic-charm component inside the proton would be an enhancement of $Z+c$ production, mainly at large values of the transverse momentum of the charm-quark jet. This is the first measurement of $\mathrm{Z}+\mathrm{c}$ in the central region.

\section{The CMS detector}

The central feature of the CMS apparatus is a superconducting solenoid of $6 \mathrm{~m}$ internal diameter, providing a magnetic field of $3.8 \mathrm{~T}$. Within the solenoid volume are a silicon pixel and strip tracker, a lead tungstate crystal electromagnetic calorimeter (ECAL), and a brass and scintillator hadron calorimeter (HCAL), each composed of a barrel and two endcap sections. Forward calorimeters extend the pseudorapidity coverage provided by the barrel and endcap detectors. Muons are detected in gas-ionization chambers embedded in the steel flux-return yoke outside the solenoid. A more detailed description of the CMS detector, together with a definition of the coordinate system used and the relevant kinematic variables, can be found in [2].

\section{W+c associated production:}

At the LHC, $\mathrm{W}+\mathrm{c}$ production is dominated by $\overline{\mathrm{q}} \mathrm{g} \rightarrow \mathrm{W}^{+}+\overline{\mathrm{c}}$ and $\mathrm{qg} \rightarrow \mathrm{W}^{-}+\mathrm{c}$ contributions at the hard scattering level. A key property of a general $\mathrm{qg} \rightarrow \mathrm{W}+\mathrm{c}$ process is the presence in the final state of a charm quark and a $\mathrm{W}$ boson with opposite sign charges (OS). Gluon splitting processes like d $\overline{\mathrm{u}} \rightarrow \mathrm{W}^{-}+\mathrm{g} \rightarrow \mathrm{W}^{-}+\mathrm{c} \overline{\mathrm{c}}$ also give rise to final states with a $\mathrm{W}$ and a charm quark, but in this case, there is an additional charm quark with the same sign (SS) as the W boson. Most of the background processes deliver evenly OS and SS events, whereas qg $\rightarrow \mathrm{W}+\mathrm{c}$ is always OS. A pure sample of c-jets can thus be obtained by statistical OS-SS subtraction. Measurements are presented of the associated production of a $W$ boson and a charm quark $(\mathrm{W}+\mathrm{c})$ in proton-proton collisions at a center-of-mass energies of 8 and $13 \mathrm{TeV}$ [3, 4]. The data correspond to integrated luminosities of 19.7 and $35.7 \mathrm{fb}^{-1}$. In all the analyses, the simulated samples are corrected for differences between data and MC description in lepton trigger, reconstruction and identification efficiencies.Correction factors are applied to the simulation as weigths on an event-by-event basis. 
The $W$ bosons are identified by their decays into a lepton (electron or muon) and a neutrino. The charm quark is identified inclusively through the semileptonic decay of a $\mathrm{c}$ flavoured hadron with a reconstructed muon inside the jet, and other inclusive charm hadron decays by reconstructing their secondary decay vertex ( $8 \mathrm{TeV}$ analysis), or exclusively by the full reconstruction of the $\mathrm{D}^{* \pm}(2010)$ meson in its decay $\mathrm{D}^{*-}(2010) \rightarrow \overline{\mathrm{D}}^{0}+\pi_{\text {slow }}^{-} \rightarrow K^{+}+\pi^{-}+\pi_{\text {slow }}^{-}$(+c.c.) (13 TeV analysis). The measurements are performed for the transverse momentum of the lepton from the $W$ boson decay greater than 30 (26) GeV in the pseudorapidity range $\left|\eta^{\mu}\right|<2.1(2.4)$ and for transverse momentum of the charm quark greater than $5 \mathrm{GeV}$ for the $8 \mathrm{TeV}(13 \mathrm{TeV})$ analysis. Measurements of the associated production of a $\mathrm{W}$ boson and a charm quark $(\mathrm{W}+\mathrm{c})$ are presented as total cross section, differential cross section as a function of the pseudorapidity and transverse momentum of the lepton from the $\mathrm{W}$ decay and the $\sigma\left(\mathrm{pp} \rightarrow \mathrm{W}^{+}+\overline{\mathrm{c}}\right) / \sigma\left(\mathrm{pp} \rightarrow \mathrm{W}^{-}+\mathrm{c}\right)$ cross section ratio both inclusively and differentially. Total cross sections of $\sigma(\mathrm{pp} \rightarrow \mathrm{W}+\mathrm{c})=116.3 \pm 0.7$ (stat.) \pm 5.2 (syst.) pb (at $8 \mathrm{TeV}$ ) and $1026 \pm 31$ (stat. ${ }_{-72}^{+76}$ (syst.) pb (at $13 \mathrm{TeV}$ ) are obtained. The measured inclusive and differential cross sections of $\mathrm{W}+\mathrm{c}$ are compared to QCD predictions at NLO $\left(\mathscr{O}\left(\alpha_{s}^{2}\right)\right)$, obtained using MCFM [5] (see Fig. 1 (a) and (b) and Fig. 2 (a)). Similar to previous measurement of W+c at $7 \mathrm{TeV}$ [6], the mass of the charm quark is chosen to be $m_{c}=1.5 \mathrm{GeV}$, and the factorization and the renormalization scales are set to the value of the $W$ boson mass. The calculation is performed for $p_{T}^{l}>30$ (26) GeV, $\left|\eta^{l}\right|<2.1$ (2.4) and $p_{T}^{c}>5 \mathrm{GeV}$ for the 8 (13) TeV analysis using different PDF sets (ABMP16nlo [7], CT14nlo [8], MMHT14nlo [9], and NNPDF3.1nlo [10]). Good agreement between NLO predictions and the measurements is observed. For the cross section ratio $\sigma\left(\mathrm{pp} \rightarrow \mathrm{W}^{+}+\overline{\mathrm{c}}\right) / \sigma\left(\mathrm{pp} \rightarrow \mathrm{W}^{-}+\mathrm{c}\right)$, all theoretical predictions are in good agreement with the measured value. The present $13 \mathrm{TeV}$ measurement of the $\mathrm{W}+\mathrm{c}$ production cross section is used in a QCD analysis because of its potential to access the strange quark distribution directly through the processes above. The measurements of a previous QCD analysis [6] are also included as well as earlier inclusive DIS measurements [11] and results from CMS on the lepton charge asymmetry in $\mathrm{W}$ production at $8 \mathrm{TeV}$ [12]. The XFITTER [13] fit framework for PDF determination is used. with the parton distributions evolved by using the Dokshitzer-Gribov-Lipatov-Altarelli-Parisi equations $[14,15,16,17,18,19]$ at NLO, as implemented in the QCDNUM 17-00/06 program [20]. The strange quark distribution and strangeness suppression factor obtained in the fit agree with results from neutrino-scattering experiments (Fig. 2 (b)).
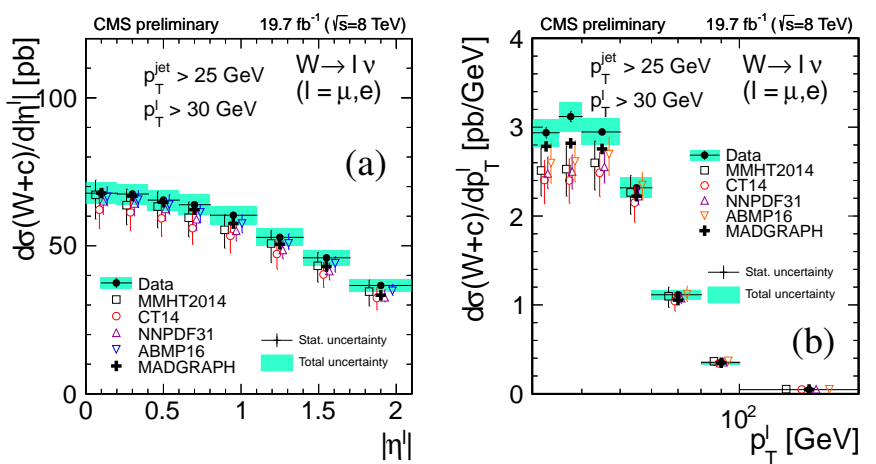

Figure 1: (a) Differential cross section of $W+c$ production as a function of (a) the pseudorapidity and (b) the transverse momentum of the lepton from the $\mathrm{W}$ decay ( $8 \mathrm{TeV}$ analysis [3]). 

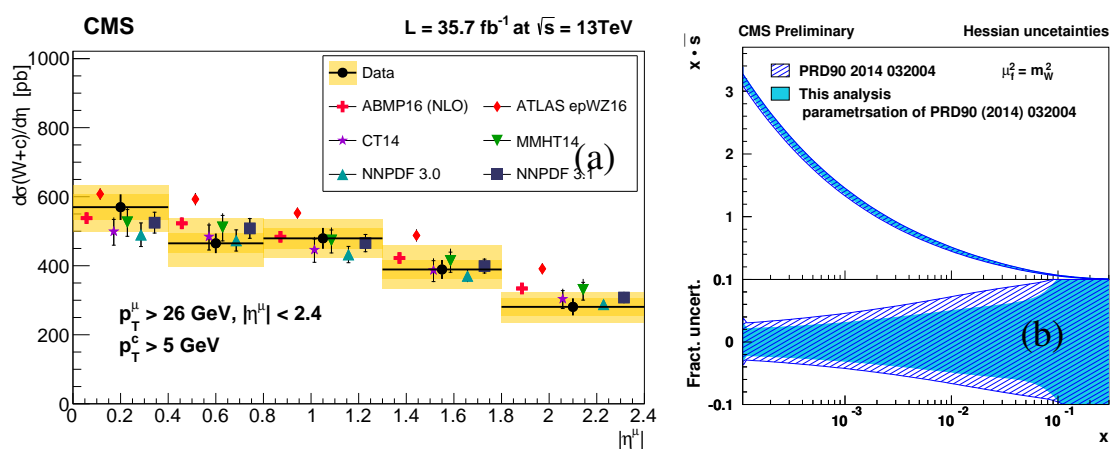

Figure 2: (a) Differential cross section of $\mathrm{W}+\mathrm{c}$ production as a function of the pseudorapidity of the muon from the $\mathrm{W}$ decay. (b) The s-quark distribution (upper panel) in the proton and its relative uncertainty (bottom panel) as functions of $x$ at the factorisation scale $m_{W}^{2}$ [4]. The PDF uncertainties resulting from the fit are shown. In the bottom panels the distributions are normalized to 1 .
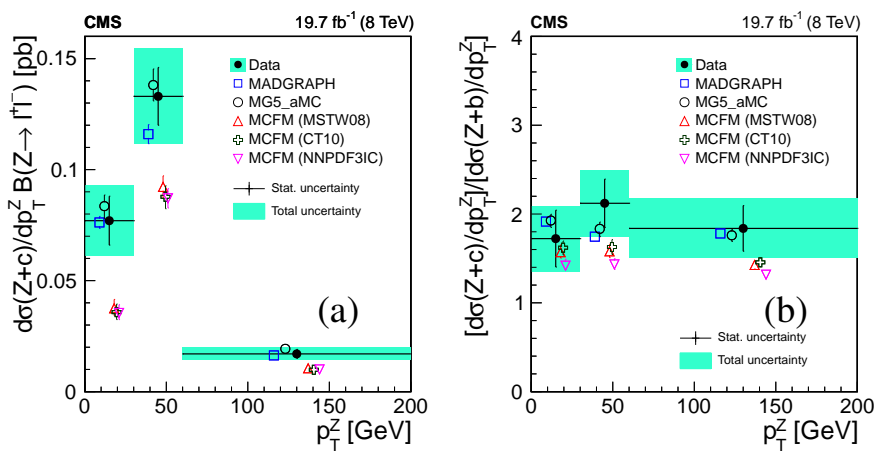

Figure 3: (a) Differential cross section of $Z+c$ production and (b) cross section $Z+b / Z+c$ ratio as a function of the transverse momentum of the $Z$ boson [21].

\section{Z+c associated production:}

A measurement of the production cross section of a $\mathrm{Z}$ boson and at least one jet originating from a c-quark $(\mathrm{Z}+\mathrm{c})$ and the cross section ratio $\sigma(\mathrm{pp} \rightarrow \mathrm{Z}+\mathrm{c}+\mathrm{X}) / \sigma(\mathrm{pp} \rightarrow \mathrm{Z}+\mathrm{b}+\mathrm{X})$ in $\mathrm{pp}$ collisions at a center-of-mass energy of $8 \mathrm{TeV}$ is presented. [21]. The analysis is conducted with a data sample corresponding to an integrated luminosity of $19.7 \mathrm{fb}^{-1}$. The Z-boson candidates are identified through their decay into a pair of leptons (electrons or muons). Jets originating from heavy flavour quarks are identified using semileptonic decays of c- or b-flavoured hadrons and hadronic decays of charm hadrons. Cross section and cross sections ratio are measured at the level of stable particles. In order to minimize acceptance corrections, the measurements are restricted to a phase space close to the experimental fiducial volume with optimized sensitivity for these processes: two leptons with $\mathrm{p}_{\mathrm{T}}^{l}>20 \mathrm{GeV}$, pseudorapidity $\left|\eta^{l}\right|<2.1$, and with the dilepton invariant mass consistent with the mass of the $\mathrm{Z}$ boson, $71<m_{l l}<111 \mathrm{GeV}$, and a c- (b-) jet with $\mathrm{p}_{\mathrm{T}}{ }^{\text {jet }}>25 \mathrm{GeV}$, pseudorapidity $\left|\eta^{\text {jet }}\right|<2.5$ and separated from the leptons from the Z-boson candidate by a distance $\Delta R($ jet,$l)=\sqrt{(\Delta \eta)^{2}+(\Delta \phi)^{2}}>0.5$.

The cross sections obtained in the electron and muon decay channels are consistent and are averaged to obtain the final cross section, thus decreasing the associated statistical uncertainty. The $\mathrm{Z}+\mathrm{c}$ production cross section is measured to be $\sigma(\mathrm{pp} \rightarrow \mathrm{Z}+\mathrm{c}+\mathrm{X})=8.6 \pm 0.5$ (stat.) \pm 0.7 (syst.) pb. The relative production of a $\mathrm{Z}$ boson and at least one c- or b-quark jet is analysed in 
terms of cross sections ratio. The ratio of the $\mathrm{Z}+\mathrm{c}$ and $\mathrm{Z}+\mathrm{b}$ production cross sections is measured to be $\sigma(\mathrm{pp} \rightarrow \mathrm{Z}+\mathrm{c}+\mathrm{X}) / \sigma(\mathrm{pp} \rightarrow \mathrm{Z}+\mathrm{b}+\mathrm{X})=2.0 \pm 0.2$ (stat.) \pm 0.2 (syst.). The $\mathrm{Z}+\mathrm{c}$ production cross section and the cross sections ratio are also measured differentially as a function of transverse momentum of the $\mathrm{Z}$ boson (Fig. 3 (a)) and of the heavy flavour jet (Fig. 3 (b)). The measurements are in agreement with the LO predictions from MADGRAPH [22] + PYTHIA6 [23] normalized to next-to-next-to-leading order (NNLO) calculation computed with FEWZ [24] and NLO predictions from MADGRAPH5_AMC@NLO [25] + PYTHIA8 [26]. Predictions from the MCFM program are lower than the measured $\mathrm{Z}+\mathrm{c}$ cross section, both inclusive and differentially. This difference can be explained by the absence of parton shower development and nonperturbative effects in the MCFM calculation. A better description is reached in terms of the $\mathrm{Z}+\mathrm{c} / \mathrm{Z}+\mathrm{b}$ cross sections ratio. Measurements in the highest $\mathrm{p}_{\mathrm{T}}{ }^{Z}\left(\mathrm{p}_{\mathrm{T}}{ }^{\text {jet }}\right)$ region analyzed, $60<\mathrm{p}_{\mathrm{T}}{ }^{Z}\left(\mathrm{p}_{\mathrm{T}}{ }^{\text {jet }}\right)<200 \mathrm{GeV}$, would be sensitive to the existence of an intrinsic charm (IC) component inside the proton if this IC component were large enough to induce a significant enhancement in the $\mathrm{Z}+\mathrm{c}$ production cross section. A $2 \%$ charm quark component (intrinsic + perturbative) would end in a 20-25\% increase in the production of $\mathrm{Z}+\mathrm{c}$ events with a $\mathrm{p}_{\mathrm{T}}{ }^{Z} \approx 100 \mathrm{GeV}$. However, our measurements of the $\mathrm{Z}+\mathrm{c}$ cross section and $(Z+c) /(Z+b)$ cross section ratio are consistent with predictions using PDF sets with no IC component.

The main uncertainty is related with the charm fractions for the production and decay of c-hadrons in the simulated samples, and with the determination of the efficiency to identify charm in jets.

In summary, the $s$ PDF obtained from the $\mathrm{W}+\mathrm{c}$ analysis is in agreement with previous measurements and we have presented the first measurement of $\mathrm{Z}+\mathrm{c}$ in the central region. A good description of the results is given by MADGRAPH5_AMC@NLO using PDF sets with no IC component. Good prospects for new results in vector boson and heavy flavor with full statistics will be offered by Run II with the more than $150 \mathrm{fb}^{-1}$ collected at $13 \mathrm{TeV}$.

\section{References}

[1] R. D. Ball, V. Bertone, M. Bonvini, S. Carrazza, S. Forte, A. Guffanti, N. P. Hartland, J. Rojo, and L. Rottoli, "A Determination of the Charm Content of the Proton," 2016.

[2] S. Chatrchyan et al., "The CMS experiment at the CERN LHC," JINST, vol. 3, p. S08004, 2008.

[3] CMS Collaboration CMS Physics Analysis Summary CMS-PAS-SMP-18-013, CERN, 2019.

[4] A.M. Sirunyan, A. Tumasyan, W. Adam et al. Eur. Phys. J. C, vol. 79, p. 269, 2018.

[5] J. M. Campbell and R. K. Ellis, "MCFM for the Tevatron and the LHC," Nucl. Phys. Proc. Suppl., vol. 205, p. 10, 2010.

[6] S. Chatrchyan et al., "Measurement of the muon charge asymmetry in inclusive $p p \rightarrow W+X$ production at $\sqrt{s}=7 \mathrm{TeV}$ and an improved determination of light parton distribution functions," Phys. Rev. D, vol. 90, p. 032004, 2014.

[7] S. Alekhin, J. Blümlein, and S. Moch, "NLO PDFs from the ABMP16 fit," tech. rep., 2018.

[8] S. Dulat, T.-J. Hou, J. Gao, M. Guzzi, J. Huston, P. Nadolsky, J. Pumplin, C. Schmidt, D. Stump, and C. P. Yuan Phys. Rev. D, vol. 93, p. 033006, 2016.

[9] L. A. Harland-Lang, A. D. Martin, P. Motylinski, and R. S. Thorne Eur. Phys. J. C, vol. 75, p. 204, 2015. 
[10] R. D. Ball et al. Eur. Phys. J. C, vol. 77, p. 663, 2017.

[11] H1 and ZEUS Collaborations, "Combination of measurements of inclusive deep inelastic $e^{ \pm} p$ scattering cross sections and QCD analysis of HERA data," Eur. Phys. J. C, vol. 75, p. 580, 2015.

[12] V. Khachatryan et al., "Measurement of the differential cross section and charge asymmetry for inclusive pp $\rightarrow \mathrm{W}^{ \pm}+X$ production at $\sqrt{s}=8 \mathrm{TeV}$, , Eur. Phys. J. C, vol. 76, p. 469, 2016.

[13] S. Alekhin et al., "HERAFitter," Eur. Phys. J. C, vol. 75, p. 304, 2015.

[14] V. N. Gribov and L. N. Lipatov, “Deep inelastic $e-p$ scattering in perturbation theory," Sov. J. Nucl. Phys., vol. 15, p. 438, 1972.

[15] G. Altarelli and G. Parisi, “Asymptotic freedom in parton language,” Nucl. Phys. B, vol. 126, p. 298, 1977.

[16] G. Curci, W. Furmanski, and R. Petronzio, "Evolution of parton densities beyond leading order: The non-singlet case," Nucl. Phys. B, vol. 175, p. 27, 1980.

[17] W. Furmanski and R. Petronzio, "Singlet parton densities beyond leading order," Phys. Lett. B, vol. 97, p. 437, 1980.

[18] S. Moch, J. A. M. Vermaseren, and A. Vogt, “The three-loop splitting functions in QCD: the non-singlet case,” Nucl. Phys. B, vol. 688, p. 101, 2004.

[19] A. Vogt, S. Moch, and J. A. M. Vermaseren, "The three-loop splitting functions in QCD: the singlet case,” Nucl. Phys. B, vol. 691, p. 129, 2004.

[20] M. Botje, "QCDNUM: fast QCD evolution and convolution,” Comput. Phys. Commun., vol. 182, p. 490, 2011.

[21] CMS Collaboration, "Measurement of associated $\mathrm{Z}+$ charm production in proton-proton collisions at $\sqrt{s}=8$ TeV," Eur. Phys. J. C, vol. 78, p. 287, 2018.

[22] J. Alwall, M. Herquet, F. Maltoni, O. Mattelaer, and T. Stelzer, "Madgraph 5 : Going beyond," JHEP, vol. 06, p. 128, 2011.

[23] T. Sjostrand, S. Mrenna, and P. Z. Skands, "PYTHIA 6.4 Physics and Manual," JHEP, vol. 05, p. 026, 2006.

[24] Y. Li and F. Petriello, "Combining QCD and electroweak corrections to dilepton production in FEWZ," Phys. Rev. D, vol. 86, p. 094034, 2012.

[25] J. Alwall, R. Frederix, S. Frixione, V. Hirschi, F. Maltoni, O. Mattelaer, H. S. Shao, T. Stelzer, P. Torrielli, and M. Zaro JHEP, vol. 07, p. 079, 2014.

[26] T. Sjostrand, S. Mrenna, and P. Z. Skands, "Introduction to PYTHIA 8.1," Comput. Phys. Commun., vol. 178, pp. 852-867, 2008. 